\title{
Precision Therapy of Pancreatic Cancer: From Bench to Bedside
}

\author{
Katrin Jana Ciecielski Alexandra Berninger Hana Algül \\ Comprehensive Cancer Center Munich (CCCM $\left.{ }^{\mathrm{TUM}}\right)$, Klinikum rechts der Isar, Technische Universität München, \\ Munich, Germany
}

\section{Keywords}

Pancreatic adenocarcinoma $\cdot$ Targeted therapy $\cdot$ KRAS · SHP2

\begin{abstract}
Background: Pancreatic ductal adenocarcinoma (PDAC), with a mortality rate of $94 \%$ and a 5-year-survival rate of only $8 \%$, is one of the deadliest cancer entities worldwide, and early diagnostic methods as well as effective therapies are urgently needed. Summary: This review summarizes current clinical procedure and recent developments of oncological therapy in the palliative setting of metastatic PDAC. It further gives examples of successful, as well as failed, targeted therapy approaches and finally discusses promising ongoing research into the decade-old question of the "undruggability" of KRAS. Key Messages: Bench-driven concepts change the clinical landscape from "one size fits all" towards precision medicine. With growing insight into the molecular mechanisms of pancreatic cancer the era of targeted therapy in PDAC is gaining a new momentum.

(c) 2020 S. Karger AG, Basel
\end{abstract}

\section{Introduction}

Pancreatic ductal adenocarcinoma (PDAC) is one of the most prevalent neoplastic diseases of the pancreas, accounting for $4.5 \%$ of all cancer-related deaths worldwide [1-3]. The dismal prognosis, with a 5-year overall survival (OS) of less than $8 \%$, is due to infrequence of typical symptoms, resulting in late diagnosis and the high poten- tial to metastasize [4]. Only 10-20\% of all PDAC patients are diagnosed with PDAC at resectable stages [5] and even where surgery is feasible, PDAC is prone to relapse and developing resistance to supportive chemotherapy, making treatment options challenging [6-9]. Another third of the patients present with locally advanced but nonmetastatic disease, and usually undergo neoadjuvant chemotherapy, with the option of radiation, followed by surgery as a possible curative treatment. In most cases, however, patients are diagnosed at the stage of locally advanced and metastatic disease where palliative chemotherapy, aiming to prolong the OS and improve quality of live, is the only remaining treatment option [3].

\section{Current Clinical Standard of Care}

Systemic chemotherapy is commonly employed as a first-line treatment for patients with nonresectable metastasizing PDAC [9]. Until 2011, gemcitabine was the only validated standard of care $[10,11]$. Since then however, FOLFIRINOX (a combination of folinic acid, fluorouracil, irinotecan, and oxaliplatin) successfully competes with gemcitabine by increasing OS by 4.3 months (OS 11.1 vs. 6.8 months; $p<0.001)[12,13]$. Unlike the combination therapy of gemcitabine plus nab-paclitaxel (gem/nabPac) established in 2013, which has a similar OS outcome to FOLFIRINOX (OS of 8.5 months compared to 6.7 months with gemcitabine only, $p<0.001)[13,14]$, mainly patients with good Eastern Cooperative Oncology Group (ECOG) performance status $(99.4 \%$ ECOG $<2)$ have been administered with FOLFIRINOX in the ACCORD trial, 


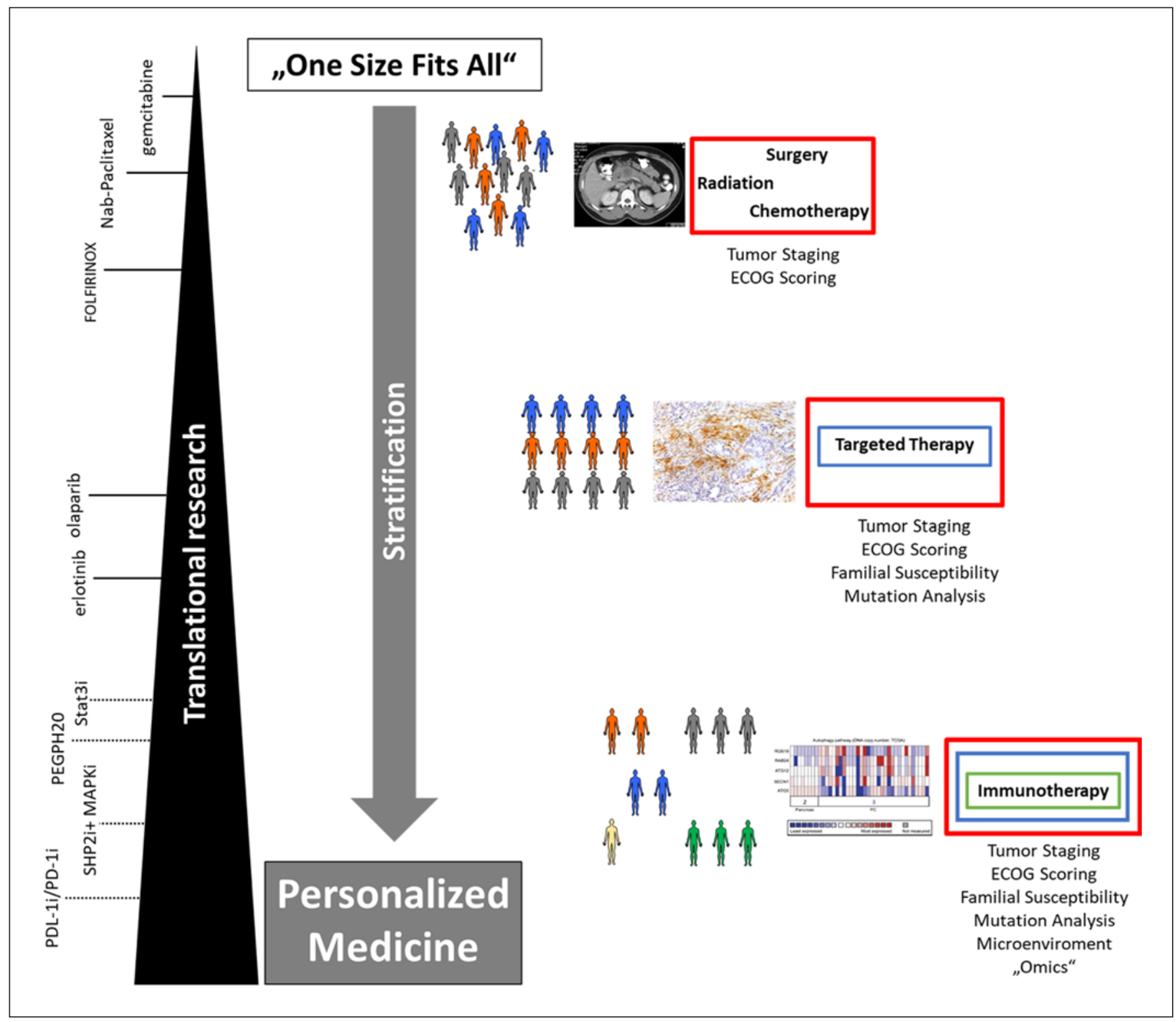

Fig. 1. Basic and translational research drives the stratification process from a "one size fits all" approach to personalized medicine.

impeding a direct comparison between the two studies [3, 11]. However, substantial side effects like febrile neutropenia, thrombocytopenia, diarrhea, sensory neuropathy, and alopecia are more commonly seen with the FOLFIRINOX regimen, suggesting FOLFIRINOX as a first-line therapy rather for younger patients with a good performance status [11, 12]. Nab-paclitaxel has demonstrated safe results in first-line treatment for elderly or weaker patients with ECOG $>1[3,15]$. Surgical patients with a good ECOG score are likely to benefit from modified FOLFIRINOX rather than gemcitabine as an adjuvant chemotherapy to contain relapse and increase OS [16].

For those patients, almost 50\% [11], who progress under first-line therapy, options for second-line treatments are available. The choice of second-line therapy depends on preceding first-line therapy, the patient's ECOG score, and prior adverse events, like polyneuropathy [13]. The standard front-line therapy gem/nab-Pac is commonly followed by a 5-FU-based regimen, like OFF (oxaliplatin, 5-FU, folinic acid), resulting in an OS of 5.9 months compared to 3.3 months in the $5-\mathrm{FU}$ control $[11,16,17]$. However, previous platin-based therapy should be replaced by 5-FU/LV/nal-IRI (OS 6.1 months) or FOLFIRI (OS 5 months) as second-line regimen in patients suffering from polyneuropathy $[13,18,19]$. The gem/nab-Pac regimen is recommended for patients who received FOLFIRINOX in the front line, provided they have a good performance status $[11,20]$. For the others, monotherapy with gemcitabine or combination of gemcitabine plus erlotinib may be an option $[13,21]$. 
To date, no standard-of-care recommendation exists for those patients who have progressed beyond two lines of systemic therapies, and enrollment in clinical trials is advisable if the ECOG score allows $[11,20]$. So far, only one phase 3 study (NAPOLI-1) for third-line therapy has been validated [13]. Within the scope of NAPOLI-1, patients received 5-FU/LV/nal-IRI after gemcitabine therapy [19]. Unfortunately, most of the patients are not in a health condition to tolerate additional third-line treatments. These patients should be provided with best supportive care at all times.

Since all chemotherapeutic strategies for PDAC show mediocre efficacy and are often associated with severe toxicity, a more targeted approach exploiting tumor-specific vulnerabilities might be advisable [11]. Significant research efforts within the past decade have led to increasing understanding of PDAC genetics, pathophysiology, and tumor biology and thus uncovered a vast array of possible targets, which are being explored in preclinical and clinical trials. In the following, we briefly summarize examples of completed and ongoing studies focusing on genomic instability, core signaling pathways, microenvironment, and oncogene dependence and thus stratify the patient population to increase treatment efficacy (Fig. 1).

\section{Targeted Therapy}

Targeting Breast Cancer-Induced Genomic Instability

A successful example, where basic "bench" research let to the definition of a distinct patient population likely to benefit from targeted treatment, is the recently conducted clinical phase 3 POLO trial. This study examined patients with a familial inherited susceptibility to pancreatic cancer by germline mutations in the tumor suppressor genes $B R C A 1 / 2$ [22], which occurs with an incidence of around $4-7 \%$ of all pancreatic cancer cases [13, 23, 24]. As has been reported for ovarian and breast cancer [23, 25], $B R C A$ loss-of function mutations cause genomic instability via disruption of the homologous recombination (HR) DNA-damage repair mechanism (HR) and thus increase the risk of PDAC formation [24]. Patients harboring the $B R C A$ mutation were shown to respond to front-line therapy with platinum-based drugs (e.g., oxaliplatin in FOLFIRINOX) $[13,26,27]$ which intercalate into the DNA, resulting in cell apoptosis due to faulty HR machinery. Research suggests that this effect can be enhanced and made clinically useful by pharmacologically targeting the base-excision repair mechanism, which is associated with the repair of DNA single-strand breaks (SSB). Indeed, shutting down poly(ADP-ribose)-polymerase (PARP), normally recruiting enzymes to heal single-strand breaks, as a "second hit" targeting DNA damage machinery, resulted in the accumulation of DNA double-strand breaks and the induction of cell death [27]. Translating these concepts to the clinical setting showed significantly improved progression-free survival (7.4 vs. 3.8 months; $p=0.004)$ in $B R C A$ mutant patients with metastatic pancreatic cancer, who received the PARP inhibitor olaparib as a maintenance therapy after first-line platinum-based chemotherapy compared to placebo [23]. Furthermore, results of the TAPUR trial (NCT02693535), reported at this year's ASCO meeting, show that olaparib monotherapy had significant antitumor activity in heavily pretreated pancreatic cancer patients with germline (disease control in $5 / 12$ patients) or somatic (disease control in 3/16 patients) BRCA1/2-inactivating mutations [28]. These findings are in agreement with results from a retrospective analysis of the Know Your Tumor (KYT) registry trial [29], which found that patients with pancreatic cancer whose tumors harbor targetable molecular alterations, like BRCA1/2 mutations, and who received molecularly matched therapy had a significantly longer median OS than similar patients who did not receive molecularly matched therapy.

\section{Targeting Epidermal Growth Factor Receptor}

Another research-driven approach is the inhibition of key regulatory pathways in the tumor and the tumor microenvironment. Scientifically sound but not yet leading to clinically beneficial results is the administration of gemcitabine combined with the epidermal growth factor receptor (EGFR) inhibitor erlotinib [30]. The receptor tyrosine kinase (RTK) EGFR is known to be involved in growth regulation, differentiation, and proliferation of different cancer types [31] and has been shown to play a significant role in the progression of PDAC [32]. Under these circumstances, a targeted therapy combined with gemcitabine seemed reasonable. However, in a first clinical trial OS was only slightly increased compared to gemcitabine alone (6.24 vs. 5.92 months; $p=0.038$ ) [33]. Notably, the patients who developed a skin rash within a median onset of 1-2 weeks of erlotinib treatment have been associated with a greater likelihood of response [34]. A clinical study showing improved OS in KRAS wild-type compared to KRAS mutant PDAC patients under erlotinib regimen (wild-type KRAS 8.0 months vs. mutant KRAS 6.6 months) [30] suggests that the patient population should be stratified with regard to EGFR activation as well as KRAS status prior to treatment.

\section{Targeting Signal Transducer and Activator of \\ Transcription 3}

The same underlying idea is driving research into the clinical application of signal transducer and activator of transcription 3 (STAT3) pathway inhibition. The STAT3 transcription factor is an important regulator of stem cell self-renewal, cancer cell survival, and inflammation [35]. 
Its activation depends on the phosphorylation of a conserved tyrosine residue (Y705) by upstream kinases like Janus kinase 2 (JAK2), which in turn gets activated through ligands like IL-6, leukemia inhibitor factor, IL11 , oncostatin $M$, ciliary neurotrophic factor, or IL-27 binding to the ubiquitously expressed co-receptor gp 130 $[36,37]$. While STAT3 is dispensable for normal pancreas development, the majority of PDAC show constitutive activation of STAT3 [35]. In 2011, our group was able to show that, in addition to mutant Kras ${ }^{\text {G12D }}$, IL-6 from the myeloid compartment activates STAT3 via trans-signaling and is necessary for the progression from PanIN formation to PDAC [36].

A later study in our group showed, furthermore, that the loss of p53 function which commonly occurs in advanced PanIN stages activates JAK2-STAT3 signaling, which not only promotes tumor growth, but also the modification of the tumor stroma as well as resistance to gemcitabine [38]. Following the hypothesis that remodeling of desmoplastic stroma by inhibiting persistent STAT3 activation would result in better delivery of chemotherapeutic agents to the cancer cells, Wörmann et al. [38] treated tumor-bearing KPC mice with a combination of the semi-selective JAK2 inhibitor fedratinib (TG101348) and gemcitabine. Compared to either single treatment, the mice receiving a combination of gemcitabine and fedratinib showed a markedly reduced rate of tumor growth as well as significantly increased OS. While the preclinical in vitro and in vivo data would suggest that the combination of JAK/STAT inhibition plus chemotherapy is beneficial to PDAC patients, translation to the clinical setting was disappointingly unsuccessful. While the phase 2 RECAP study, combining the JAK $1 / 2$ inhibitor ruxolitinib with capecitabine, in gemcitabinerefractory metastatic PDAC patients showed improved OS in a subgroup of patients with significantly elevated CRP levels [11,39], two subsequent phase 3 studies, JANUS1 (NCT02117479) and JANUS2 (NCT02119663), with the same drug combination in a selected population with high modified Glasgow Prognostic Scores were discontinued due to lack of efficacy [11, 40]. Although the clinical trials were based on sound scientific reasoning, the lack of efficacy observed in the JANUS 1 and 2 trials, as well as studies combining momelotinib and chemotherapy (NCT02244489), highlights the complexity of the JAK/STAT pathway, tumor inflammation in pancreatic cancer, and the need for better patient selection [40].

Targeting Hyaluronic Acid in the Extracellular Matrix

Further translational research projects focus on compartment-specific drug treatment in PDAC, as tumor progression is related to interaction between cancer cells and the surrounding microenvironment [41]. The microenvironment of pancreatic cancer dominates tumor mass and comprises extracellular matrix, fibroblasts, immune cells, and endothelial cells resulting in a dense and poorly perfused desmoplastic stroma [41] and is conceived as a major barrier to effective chemotherapy. The concept behind the phase 3 HALO 301 trial was to decrease the tumor mass and vascular constriction by depleting hyaluronic acid (HA) in the extracellular matrix, in order to facilitate tumor perfusion with gemcitabine or nab-paclitaxel $[42,43]$. By a disbalance in synthesis and degradation, HA has been reported to accumulate in a malignant cancer environment, leading to enhanced cell migration, adhesion, and proliferation $[41,44]$. Although the study was based on a sound scientific rationale, taking into account that high levels of HA correlate with poor patient outcome [45], as well as promising preclinical and early clinical results, HALO 301 failed in late phase 3 missing its primary endpoint [43]. Indeed, attempts of explanation shed light on the contrary role of desmoplastic stroma in tumor tissue. On the one hand, stroma may be a physical barrier for drug delivery, while on the other hand it may be protective due to restriction of aggressive tumor evasion [42]. Moreover, immunosuppressive cells like myeloid-derived suppressor cells and regulatory $\mathrm{T}$ cells are also localized in the tumor microenvironment and play a pivotal role in immunosurveillance of PDAC [42]. Due to that fact, a modified clinical study with the HA drug PEGPH20 in combination with pembrolizumab (PD-1 inhibitor) is currently under investigation to analyze the relation between desmoplastic depletion and sensitivity to immune therapy [46].

Although three of the four examples on targeted therapies discussed above failed to generate significant clinical benefit to PDAC patients, they have proven the necessity for close collaboration between basic and clinical research.

\section{Targeting the "Undruggable" KRAS}

$R A S$ genes (HRAS, KRAS, and NRAS), encoding proteins of the small GTPase family that drive essential cellular processes like survival, proliferation, or differentiation [11], constitute the most frequently mutated oncogene family in human cancer. Mutant KRAS is the predominant isoform in cancer and the only one mutated in PDAC. KRAS mutations are found in about $94 \%$ of all PDAC cases, making it arguably the most RAS-addicted cancer type [47-52]. More than $90 \%$ of the KRAS mutations in PDAC are found in codon 12, resulting in an amino acid substitution of glycine $(\mathrm{G})$ to arginine $(\mathrm{R})$, aspartic acid (D), or valine $(\mathrm{V})[11,52]$. These alterations render the molecule insensitive to GTPase-activating proteins (RAS-GAPs), resulting in pronounced attenuation of intrinsic GTP hydrolysis and a prolonged GTP-bound activated state $[11,53-55]$. Due to substantial experimental evidence showing the essential role of mutant KRAS in 
PDAC growth, the National Cancer Institute identified targeting KRAS as one of four major priorities for pancreatic cancer research [52]. For decades, however, despite intensive research effort, no clinically effective pharmacologic RAS inhibitors were found, prompting the widely held perception that RAS proteins are "undruggable" [53]. Very recently, however, both Mirati Therapeutics and Amgen reported major breakthroughs in the field. The companies unveiled $\mathrm{KRAS}^{\mathrm{G} 12 \mathrm{C}}$-specific inhibitors - MRTX849 and AMG 510, respectively - that exhibit significant in vivo anti-tumor efficacy in mice as well as clinical activity in patients with lung and colon adenocarcinomas [56-58], and are currently under evaluation in 6 clinical trials (NCT04380753, NCT03600883, NCT04303780, NCT04185883, NCT03785249, NCT04330664). Both inhibitors gain selectivity by covalently binding to the aberrant cysteine residue found in KRAS ${ }^{\mathrm{G} 12 \mathrm{C}}$ but not the wild-type or the other mutant variants $[58,59]$. Although these inhibitors are a definite breakthrough for the therapy of tens of thousands of cancer patients, with KRAS ${ }^{\mathrm{G} 12 \mathrm{C}}$ being present in approximately $13 \%$ of lung adenocarcinoma, $3 \%$ of colorectal cancer, and $2 \%$ of the cases of other solid tumors [60], there are two major issues which dampen the success of these new inhibitors. The first and in terms of this review more important issue is the fact that, out of the $94 \%$ of PDAC cases harboring a KRAS mutation, only about $1 \%$ are $K R A S^{G 12 C}$ [52]. Thus, the vast majority of PDAC patients do not benefit from these new drugs. However, a direct KRAS inhibitor might still be on the horizon for these patients since newly developed pan-RAS inhibitors like BI 1701963, targeting KRAS:SOS1 interaction, or the RNA-based cancer vaccine mRNA-5671 are being tested in clinical phase 1 trials (NCT04111458, NCT03948763) as monotherapy or as combination therapy with MEK- or PD-1-targeted compounds, respectively [61]. The second issue dampening the success of AMG510 and MRTX849 is the fact that there are already studies indicating that $\mathrm{KRAS}^{\mathrm{G} 12 \mathrm{C}}$-specific inhibitors, as monotherapy, are unable to eradicate all cancer cells within a tumor and are likely to induce adaptive mechanisms leading to resistant cells $[62,63]$. The idea that the KRAS pathway should be targeted via a two (or more)-pronged approach is a logical step based on previous studies. Many trials targeting the RAS pathway via its effector pathways RAF-MEK-ERK or PI3K-AKT-mTOR using monotherapy were conducted and failed in the clinical setting due to the complexity of the RAS signaling network and the activation of compensatory or resistance mechanisms [11]. Our group, together with researchers from the Netherlands Cancer Institute (NKI) in Amsterdam recently published promising preclinical data about the combination of MEK and SHP2 inhibition. This two-pronged approach inhibits both the signal transfer from RTKs upstream of KRAS, as well as the signal transmission from MEK to ERK and further downstream effectors [64, 65].SHP2, encoded by PTPN11, is an ubiquitously expressed nonreceptor protein tyrosine phosphatase involved in signal transduction downstream of multiple growth factor, cytokine and integrin receptors [66], for which we were able to demonstrate a central and indispensable role in KRAS-driven tumors. Genetic deletion of Ptpn11 profoundly inhibited KRASdriven carcinogenesis in murine models of PDAC and NSCLC and delayed the progression in established tumors. Genetic deletion or pharmacological inhibition of SHP2 alone, however, was not sufficient to achieve tumor regression [64]. In vitro drug screening combining SHP2 inhibition with both classical chemotherapeutic agents (gemcitabine, paclitaxel, oxaliplatin) as well as targeted compounds against MEK, EGFR, or PI3K revealed a superior synergistic efficacy with the combination of SHP2 plus MEK inhibition. This synergism was confirmed in vivo, resulting in sustained tumor growth control in murine and human patient-derived organoids (PDX) and xenograft models of KRAS mutant PDAC and NSCLC $[64,65]$. With the concept of targeting KRAS-driven cancers via simultaneous up- and downstream inhibition, our group and Mainardi et al. [65] have shown that "at the bench" is now being translated to the bedside, and combination therapies against SHP2 and MEK (NCT03989115) or even SHP2 and KRAS $^{\mathrm{G} 12 \mathrm{C}}$ (NCT04330664) are being tested in phase $1 / 2$ clinical trials. Following this concept, the phase 1/1b SHERPA (SHP2 and ERK in Pancreatic Cancer) trial will be conducted to evaluate the tolerability and efficacy of a combination therapy using the allosteric SHP2 inhibitor RMC-4630 (provided by Revolution Medicine) and the ERK inhibitor LY3214996 (provided by Lilly). The target population are patients with metastatic KRAS mutant PDAC. Pending the results, such bench-driven concepts may lead to phase 2 and 3 trials eventually, providing the first targeted combination therapy for over $90 \%$ of PDAC patients carrying an activating mutation in the KRAS oncogene.

\section{Conclusion}

Although novel chemotherapy combinations and/or formulations, such as FOLFIRINOX, gemcitabine plus nab-paclitaxel, and liposomal irinotecan, have significantly improved pharmacotherapeutic management of PDAC and led to substantial clinical benefits, the overall prognosis for PDAC patients remains dismal. The current approach, basing treatment decisions primarily on tumor stage and overall health status, bears the risk of oversimplifying the complex reality of inter- and intratumoral heterogeneity found in PDAC. Since cancer devel- 
opment is based on acquired genetic alterations, a better understanding of the patient's mutational landscape and its relevance for treatment success is needed. As seen in the case of BRCA mutations which convey susceptibility towards PARP inhibitors, genetic screening prior to treatment allows for the identification of distinct patient populations and more precise therapy. Pishvaian et al. [29] showed that the median OS of patients with advanced PDAC harboring targetable genetic alterations and receiving matched therapy is 1 year longer than of those with actionable alterations receiving unmatched therapy, or those without targetable alterations. No other therapeutic modality had an impact of this magnitude, showing that molecular profiling together with matched targeted therapy is clearly the most promising clinical directive.

However encouraging the current research efforts are, the retrospective analysis of the American KYT program [29] shows that the feasibility of precision medicine is not only a question of identifying the correct molecular targets. Genetic profiling of (pancreatic) cancer patients is a resource-intensive endeavor, which can be very challenging for the respective health care system. Furthermore, although about $25 \%$ of the PDAC patients harbor alterations for which matched therapies are currently available, less than $5 \%$ actually receive these matched therapies due to either the aggressiveness of the disease or logistical and economic issues [29]. The future of precision medicine thus hinges on two factors: (1) identification of molecular targets and the development of matched therapies, and (2) quicker, cheaper, and more widely available molecular diagnostics. A major success regarding point 1 would be the development of either direct KRAS-targeted therapies not limited to KRAS ${ }^{\mathrm{G} 12 \mathrm{C}}$ or indirect KRAS pathway in- hibitors which do not induce resistance mechanisms. So far, indirect attempts to target the KRAS pathway, e.g., via a single RTK (like EGFR) upstream of KRAS or a single downstream effector (like MEK), were not sufficient to impede bypassing effects by the upregulation of other pathways [66], and monotherapeutic approaches with erlotinib or trametinib, respectively, have failed in clinical PDAC trials due to the onset of resistance and disease progression. We are optimistic that current researchdriven concepts like the SHERPA trial, using combination therapies that target two or more components of the KRAS signaling pathway, may be able to overcome these limitations. This treatment strategy could drastically increase the percentage of patients receiving molecularly matched therapies. Success in a bigger patient population might subsequently lead to more widespread availability of precision medicine and thus indirectly address the abovementioned point 2 .

\section{Conflict of Interest Statement}

Katrin Jana Ciecielski, Alexandra Berninger, and Hana Algül declare they have no conflicts of interest directly relevant to the content of this article.

\section{Funding Sources}

No funding was received for the preparation of this article.

\section{Author Contributions}

K.J.C. and A.B. wrote the manuscript and prepared the figure. H.A. edited and supervised the manuscript.

\section{References}

1 Global cancer observatory: cancer today [2020 May 4]. Lyon: International Agency of Research on Cancer. Available from: https:// gco.iarc.fr/today.

2 Bray F, Ferlay J, Soerjomataram I, Siegel RL, Torre LA, Jemal A. Global cancer statistics 2018: GLOBOCAN estimates of incidence and mortality worldwide for 36 cancers in 185 countries. CA Cancer J Clin. 2018 Nov;68(6): 394-424.

3 Schulz D, Algül H. Chemotherapy of pancreatic cancer in patients with poor performance. Chin Clin Oncol. 2019 Oct;8(S1):S22.

4 Siegel RL, Miller KD, Jemal A. Cancer statistics, 2016. CA Cancer J Clin. 2016 Jan-Feb; 66(1):7-30.

5 Sheahan AV, Biankin AV, Parish CR, Khachigian LM. Targeted therapies in the management of locally advanced and metastatic pancreatic cancer: a systematic review. Oncotarget. 2018 Apr;9(30):21613-27.
6 Amrutkar M, Gladhaug IP. Pancreatic cancer chemoresistance to gemcitabine. Cancers (Basel). 2017 Nov;9(11):1-23.

7 Grasso C, Jansen G, Giovannetti E. Drug resistance in pancreatic cancer: impact of altered energy metabolism. Crit Rev Oncol Hematol. 2017 Jun;114:139-52.

8 Morrison AH, Byrne KT, Vonderheide RH. Immunotherapy and Prevention of Pancreatic Cancer. Trends Cancer. 2018 Jun;4(6): 418-28.

9 Orth M, Metzger P, Gerum S, Mayerle J, Schneider G, Belka C, et al. Pancreatic ductal adenocarcinoma: biological hallmarks, current status, and future perspectives of combined modality treatment approaches. Radiat Oncol. 2019 Aug;14(1):141.
10 Burris HA 3rd, Moore MJ, Andersen J, Green MR, Rothenberg ML, Modiano MR. Improvements in survival and clinical benefit with gemcitabine as first-line therapy for patients with advanced pancreas cancer: a randomized trial. J Clin Oncol. 1997 Jun;15(6): 2403-13.

11 Ruess DA, Görgülü K, Wörmann SM, Algül H. Pharmacotherapeutic Management of Pancreatic Ductal Adenocarcinoma: Current and Emerging Concepts. Drugs Aging. 2017 May;34(5):331-57.

12 Conroy T, Desseigne F, Ychou M, Bouché O, Guimbaud R, Bécouarn Y, et al.; Groupe Tumeurs Digestives of Unicancer; PRODIGE Intergroup. FOLFIRINOX versus gemcitabine for metastatic pancreatic cancer. $\mathrm{N}$ Engl J Med. 2011 May;364(19):1817-25.

13 Abbassi R, Algül H. Palliative chemotherapy in pancreatic cancer-treatment sequences. Transl Gastroenterol Hepatol. 2019 Aug;4:56. 
14 Von Hoff DD, Ervin T, Arena FP, Chiorean EG, Infante J, Moore M, et al. Increased survival in pancreatic cancer with nab-paclitaxel plus gemcitabine. N Engl J Med. 2013 Oct; 369(18):1691-703.

15 Macarulla T, Pazo-Cid R, Guillén-Ponce C, López R, Vera R, Reboredo M, et al. Phase I/ II Trial to Evaluate the Efficacy and Safety of Nanoparticle Albumin-Bound Paclitaxel in Combination With Gemcitabine in Patients With Pancreatic Cancer and an ECOG Performance Status of 2. J Clin Oncol. 2019 Jan; 37(3):230-8.

16 Pelzer U, Schwaner I, Stieler J, Adler M, Seraphin J, Dörkenet B, et al. Best supportive care (BSC) versus oxaliplatin, folinic acid and 5-fluorouracil (OFF) plus BSC in patients for second-line advanced pancreatic cancer: a phase III-study from the German CONKOstudy group. Eur J Cancer. 2011;47(11):167681.

17 Oettle H, Riess H, Stieler JM, Heil G, Schwaner I, Seraphin J, et al. Second-line oxaliplatin, folinic acid, and fluorouracil versus folinic acid and fluorouracil alone for gemcitabine-refractory pancreatic cancer: outcomes from the CONKO-003 trial. I Clin Oncol. 2014 Aug;32(23):2423-9.

18 Zaniboni A, Aitini E, Barni S, Ferrari D, Cascinu S, Catalano V, et al. FOLFIRI as secondline chemotherapy for advanced pancreatic cancer: a GISCAD multicenter phase II study. Cancer Chemother Pharmacol. 2012 Jun; 69(6):1641-5

19 Wang-Gillam A, Li CP, Bodoky G, Dean A, Shan YS, Jameson G, et al.; NAPOLI-1 Study Group. Nanoliposomal irinotecan with fluorouracil and folinic acid in metastatic pancreatic cancer after previous gemcitabine-based therapy (NAPOLI-1): a global, randomised, open-label, phase 3 trial. Lancet. 2016 Feb; 387(10018):545-57.

20 Teague A, Lim KH, Wang-Gillam A. Advanced pancreatic adenocarcinoma: a review of current treatment strategies and developing therapies. Ther Adv Med Oncol. 2015 Mar;7(2):68-84.

21 da Rocha Lino A, Abrahão CM, Brandão RM, Gomes JR, Ferrian AM, Machado MC, et al. Role of gemcitabine as second-line therapy after progression on FOLFIRINOX in advanced pancreatic cancer: a retrospective analysis. J Gastrointest Oncol. 2015 Oct;6(5):511-5.

22 Leung K, Saif MW. BRCA-associated pancreatic cancer: the evolving management. JOP. 2013 Mar;14(2):149-51.

23 Golan T, Hammel P, Reni M, Van Cutsem E, Macarulla T, Hall MJ, et al. Maintenance olaparib for germline BRCA-mutated metastatic pancreatic cancer. N Engl J Med. 2019 Jul;381(4):317-27.

24 Kowalewski A, Szylberg Ł, Saganek M, Napiontek W, Antosik P, Grzanka D. Emerging strategies in BRCA-positive pancreatic cancer. J Cancer Res Clin Oncol. 2018 Aug; 144(8):1503-7.

25 Welcsh PL, King MC. BRCA1 and BRCA2 and the genetics of breast and ovarian cancer. Hum Mol Genet. 2001 Apr;10(7):705-13.
26 Aung KL, Holter S, Borgida A, Connor A, Pintilie M, Dhani NC, et al. Overall survival of patients with pancreatic adenocarcinoma and BRCA1 or BRCA2 germline mutation. J Clin Oncol. 2016 May;34(15_suppl):4123.

27 O'Sullivan CC, Moon DH, Kohn EC, Lee JM. Beyond breast and ovarian cancers: PARP inhibitors for BRCA mutation-associated and BRCA-like solid tumors. Front Oncol. 2014 Feb;4:1-13.

28 Ahn ER, et al. Olaparib (O) in patients (pts) with pancreatic cancer with BRCA $1 / 2$ inactivating mutations: Results from the Targeted Agent and Profiling Utilization Registry (TAPUR) study. J Clin Oncol. 2020;38(15_suppl): 4637. Available from: https://doi.org/10.1200/ JCO.2020.38.15_suppl.4637.

29 Pishvaian MJ, Blais EM, Brody JR, Lyons E, DeArbeloa P, Hendifar A, et al. Overall survival in patients with pancreatic cancer receiving matched therapies following molecular profiling: a retrospective analysis of the Know Your Tumor registry trial. Lancet Oncol. 2020 Apr;21(4):508-18.

30 Boeck S, Jung A, Laubender RP, Neumann J, Egg R, Goritschan C, et al. EGFR pathway biomarkers in erlotinib-treated patients with advanced pancreatic cancer: translational results from the randomised, crossover phase 3 trial AIO-PK0104. Br J Cancer. 2013 Feb; 108(2):469-76.

31 Guo M, Luo G, Liu C, Cheng H, Lu Y, Jin K, et al. The Prognostic and Predictive Role of Epidermal Growth Factor Receptor in Surgical Resected Pancreatic Cancer. Int J Mol Sci. 2016 Jul;17(7):1090.

32 Ueda S, Ogata S, Tsuda H, Kawarabayashi N, Kimura M, Sugiura Y, et al. The correlation between cytoplasmic overexpression of epidermal growth factor receptor and tumor aggressiveness: poor prognosis in patients with pancreatic ductal adenocarcinoma. Pancreas. 2004 Jul;29(1):e1-8.

33 Moore MJ, Goldstein D, Hamm J, Figer A, Hecht JR, Gallinger S, et al.; National Cancer Institute of Canada Clinical Trials Group. Erlotinib plus gemcitabine compared with gemcitabine alone in patients with advanced pancreatic cancer: a phase III trial of the National Cancer Institute of Canada Clinical Trials Group. J Clin Oncol. 2007 May;25(15): 1960-6.

34 Wacker B, Nagrani T, Weinberg J, Witt K, Clark G, Cagnoni PJ. Correlation between development of rash and efficacy in patients treated with the epidermal growth factor receptor tyrosine kinase inhibitor erlotinib in two large phase III studies. Clin Cancer Res. 2007 Jul;13(13):3913-21.

35 Corcoran RB, Contino G, Deshpande V, Tzatsos A, Conrad C, Benes CH, et al. STAT3 plays a critical role in KRAS-induced pancreatic tumorigenesis. Cancer Res. 2011 Jul; 71(14):5020-9.

36 Lesina M, Kurkowski MU, Ludes K, RoseJohn S, Treiber M, Klöppel G, et al. Stat3/ Socs 3 activation by IL- 6 transsignaling promotes progression of pancreatic intraepithelial neoplasia and development of pancreatic cancer. Cancer Cell. 2011 Apr;19(4):456-69.
37 Lesina M, Wörmann SM, Neuhöfer P, Song L, Algül H. Interleukin-6 in inflammatory and malignant diseases of the pancreas. Semin Immunol. 2014 Feb;26(1):80-7.

38 Wörmann SM, Song L, Ai J, Diakopoulos KN, Kurkowski MU, Görgülü K, et al. Loss of P53 Function Activates JAK2-STAT3 Signaling to Promote Pancreatic Tumor Growth, Stroma Modification, and Gemcitabine Resistance in Mice and Is Associated With Patient Survival. Gastroenterology. 2016 Jul;151(1):180-193. e12.

39 Hurwitz HI, Uppal N, Wagner SA, Bendell JC Beck JT, Wade SM 3rd, et al. Randomized, double-blind, phase II study of ruxolitinib or placebo in combination with capecitabine in patients with metastatic pancreatic cancer for whom therapy with gemcitabine has failed. J Clin Oncol. 2015 Dec;33(34):4039-47.

40 Hurwitz H, Van Cutsem E, Bendell J, Hidalgo M, Li CP, Salvo MG, et al. Ruxolitinib + capecitabine in advanced/metastatic pancreatic cancer after disease progression/intolerance to first-line therapy: JANUS 1 and 2 randomized phase III studies. Invest New Drugs. 2018 Aug;36(4):683-95.

41 Sato N, Cheng XB, Kohi S, Koga A, Hirata K. Targeting hyaluronan for the treatment of pancreatic ductal adenocarcinoma. Acta Pharm Sin B. 2016 Mar;6(2):101-5.

42 Hakim N, Patel R, Devoe C, Saif MW. Why HALO 301 Failed and Implications for Treatment of Pancreatic Cancer. Pancreas Open J. 2019;3(1):e1-e4. Available from: https://doi. org/10.17140/POJ-3-e010.

43 Tucker N. PEGH20 Combination Misses Primary Endpoint in Phase III HALO-301 Trial. 2019. Available from: https://www.targetedonc.com/view/pegh20-combination-misses-primary-endpoint-in-phase-iii-halo301trial.

44 Misra S, Hascall VC, Markwald RR, Ghatak S. Interactions between Hyaluronan and Its $\mathrm{Re}$ ceptors (CD44, RHAMM) Regulate the Activities of Inflammation and Cancer. Front Immunol. 2015 May;6(MAY):201.

45 Cheng XB, Sato N, Kohi S, Yamaguchi K. Prognostic impact of hyaluronan and its regulators in pancreatic ductal adenocarcinoma. PLoS One. 2013 Nov;8(11):e80765.

46 Chiorean EG, et al. PCRT16-001: Phase II study of PEGPH20 plus pembrolizumab for patients (pts) with hyaluronan (HA)-high refractory metastatic pancreatic ductal adenocarcinoma (mPDA). J Clin Oncol. 2020;38(4 suppl):TPS785. Available from: https://doi. org/10.1200/JCO.2020.38.4_suppl.TPS785.

47 Jones S, et al. Core signaling pathways in human pancreatic cancers revealed by global genomic analyses. Science. 2008 Sep;321(5897): 1801-6. Available from: https://doi. org/10.1126/science.1164368.

48 Biankin AV, Waddell N, Kassahn KS, Gingras MC, Muthuswamy LB, Johns AL, et al.; Australian Pancreatic Cancer Genome Initiative. Pancreatic cancer genomes reveal aberrations in axon guidance pathway genes. Nature. 2012 Nov;491(7424):399-405. 
49 Sausen M, Phallen J, Adleff V, Jones S, Leary RJ, Barrett MT, et al. Clinical implications of genomic alterations in the tumour and circulation of pancreatic cancer patients. Nat Commun. 2015 Jul;6(1):7686

50 Waddell N, Pajic M, Patch AM, Chang DK, Kassahn KS, Bailey P, et al.; Australian Pancreatic Cancer Genome Initiative. Whole genomes redefine the mutational landscape of pancreatic cancer. Nature. 2015 Feb; 518(7540):495-501.

51 Witkiewicz AK, McMillan EA, Balaji U, Baek G, Lin WC, Mansour J, et al. Whole-exome sequencing of pancreatic cancer defines genetic diversity and therapeutic targets. Nat Commun. 2015 Apr;6(1):6744.

52 Waters AM, Der CJ. KRAS: the critical driver and therapeutic target for pancreatic cancer. Cold Spring Harb Perspect Med. 2018 Sep; 8(9):a031435

53 Cox AD, Fesik SW, Kimmelman AC, Luo J, Der CJ. Drugging the undruggable RAS: mission possible? Nat Rev Drug Discov. 2014 Nov;13(11):828-51.
54 Prior IA, Lewis PD, Mattos C. A comprehensive survey of Ras mutations in cancer. Cancer Res. 2012 May;72(10):2457-67.

55 Pylayeva-Gupta Y, Grabocka E, Bar-Sagi D. RAS oncogenes: weaving a tumorigenic web. Nat Rev Cancer. 2011 Oct;11(11):761-74.

56 Canon J, Rex K, Saiki AY, Mohr C, Cooke K, Bagal D, et al. The clinical KRAS(G12C) inhibitor AMG 510 drives anti-tumour immunity. Nature. 2019 Nov;575(7781):217-23.

57 Hallin J, Engstrom LD, Hargis L, Calinisan A, Aranda R, Briere DM, et al. The KRASG12C inhibitor MRTX849 provides insight toward therapeutic susceptibility of KRAS-mutant cancers in mouse models and patients. Cancer Discov. 2020 Jan;10(1):54-71.

58 Bar-Sagi D, Knelson EH, Sequist LV. A bright future for KRAS inhibitors. Nat Can. 2020; $1(1): 25-7$.

59 Ostrem JM, Peters U, Sos ML, Wells JA, Shokat KM. K-Ras(G12C) inhibitors allosterically control GTP affinity and effector interactions. Nature. 2013 Nov;503(7477):548-51.

60 The AACR Project GENIE Consortium. AACR Project GENIE: Powering Precision Medicine through an International Consortium. Cancer Discov. 2017 Aug;7(8):818-31.
61 Mullard A. Cracking KRAS. Nat Rev Drug Discov. 2019 Nov; 18(12):887-91.

62 Hata AN, Shaw AT. Resistance looms for KRASG12C inhibitors. Nat Med. 2020 Feb; 26(2):169-70.

63 Xue JY, Zhao Y, Aronowitz J, Mai TT, Vides A, Qeriqi B, et al. Rapid non-uniform adaptation to conformation-specific KRAS(G12C) inhibition. Nature. 2020 Jan;577(7790): 421-5.

64 Ruess DA, Heynen GJ, Ciecielski KJ, Ai J, Berninger A, Kabacaoglu D, et al. Mutant KRASdriven cancers depend on PTPN11/SHP2 phosphatase. Nat Med. 2018 Jul;24(7):95460.

65 Mainardi S, Mulero-Sánchez A, Prahallad A, Germano G, Bosma A, Krimpenfort P, et al. $\mathrm{SHP} 2$ is required for growth of KRAS-mutant non-small-cell lung cancer in vivo. Nat Med. 2018 Jul;24(7):961-7.

66 Neel BG, Gu H, Pao L. The 'Shp'ing news: SH2 domain-containing tyrosine phosphatases in cell signaling. Trends Biochem Sci. 2003 Jun; 28(6):284-93. 